\title{
ADOPCIÓN DE LAS TECNOLOGÍAS INFOCOMUNICACIONALES (TI) EN DOCENTES: ACTUALIZANDO ENFOQUES.
}

Resumen: El éxito/fracaso de una innovación educativa depende en gran parte de la forma en que los actores educativos interpretan, redefinen, filtran y dan forma al cambio propuesto. La expansión de la educación hacia nuevos formatos virtuales supone que las concepciones pedagógicas se hallen desfasadas, generando un desequilibrio entre el conocimiento informático y el uso pedagógico de las TI.

Este trabajo realiza una revisión teórica de los diferentes modelos que abordan el proceso de adopción de TI en contextos educativos por parte de los docentes. Bajo el supuesto que el resultado de la interacción entre los docentes y las TI no depende de las formas en que se presentan las máquinas sino de cómo los docentes forjan una dinámica relacional que es acorde con sus creencias, se propone un esquema de adopción tecnológica de tres etapas con dos fases de transición entre ellas. El nuevo planteamiento pretende por un lado dinamizar y actualizar los enfoques tradicionales además de disipar dudas conceptuales en relación a la tecnología y los “usos” pedagógicos.

Palabras claves: Adopción tecnológica docente, teoría del comportamiento planificado, difusión de innovaciones, integración curricular tecnológica.

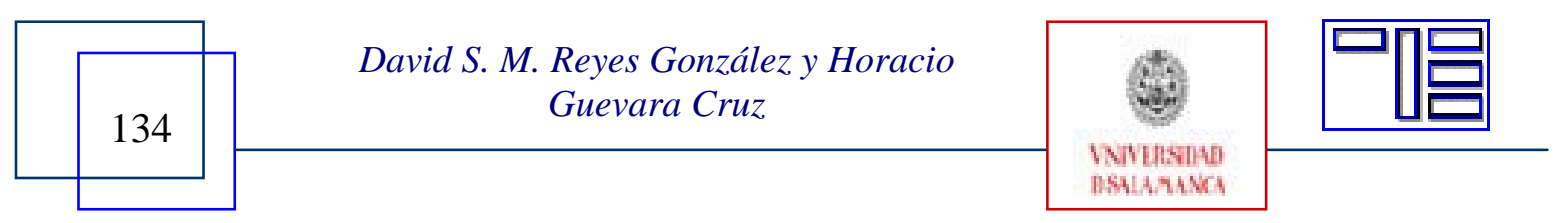




\title{
INFOCOMMUNICATIONAL TECHNOLOGY ADOPTION: RENEW AP- PROACHES.
}

\begin{abstract}
The educative innovation success/failure mainly depends in a big part of the way which educative entities represent, redefine, leak and give the shape to the proposed change. The expansion of the education toward new virtual formats, imply that the pedagogical conceptions be out of phase, which imply an unbalance between the informatical knowledgment and the pedagogical IT use.
\end{abstract}

This work do a theoric review of the differents models which are part of an IT adoption process in educational contexts in the teachers part. Under the supposed that the interaction result between the teachers and the IT do not depend of the way that the machines are presented but in how the teachers make a relational dynamism which is in compliance with its beliefs, a technological diagram is proposed of three levels with two transition phases between each them. The new planning pretends by one side to invigorate and to update the traditional focuses, besides being dispelled the conceptual doubts when we reffer to the technology and the pedagogical "uses".

Key words: Educational technological adoption, theory of the planning behaviour, innovation diffusion, technological curricular integration.

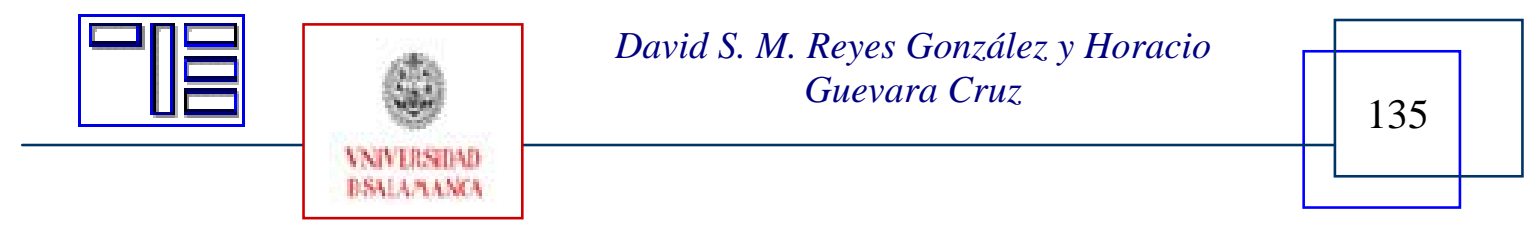




\section{ADOPTION DE LA TECHNOLOGIES DE L'INFORMATION ET DE LA COMMUNICATION DANS LES ENSEIGNANTS: MISE À JOUR DES AP- PROCHES.}

Sommaire: Le succès/échec d'une innovation éducative dépend en grande partie de la manière dont les acteurs éducatifs interprètent, redéfinissent, filtrent et donnent forme au changement proposé. L'expansion de l'éducation vers de nouveaux formats virtuels implique que les conceptions pédagogiques soient déphasées, ce qui implique un déséquilibre entre la connaissance informatique et l'utilisation pédagogique des TI.

Ce travail effectue une révision théorique des différents modèles qui abordent le processus d'adoption des TI dans des contextes éducatifs venant des enseignants. Sous l'hypothèse que le résultat de l'interaction entre les enseignants et les TI ne dépend pas des manières dans lesquelles on présente les machines mais de comment les enseignants forgent une dynamique relationnelle qui est en accord avec ses croyances, nous proposerons un schéma d'adoption technologique de trois étapes avec deux phases de transition. D’une part, la nouvelle approche prétend dynamiser et mettre à jour les analyses traditionnelles, d'autre part de dissiper des doutes conceptuels quant à la technologie et aux « usages » pédagogiques

Mots-clés: d'enseignement technologique adoption, théorie du comportement planifié, diffusion de l'innovation, scolaire intégration technologique.

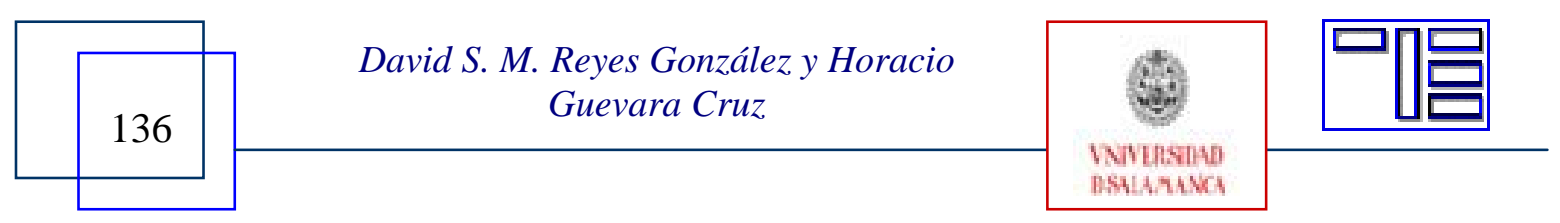




\section{ADOPCIÓN DE LAS TECNOLOGÍAS INFOCOMUNICACIONALES (TI) EN DOCENTES: ACTUALIZANDO ENFOQUES.}

David S. M. Reyes González (dsmreyes@usal.es)

Universidad Metropolitana de Ciencias de la Educación. Santiago de Chile.

Horacio Guevara Cruz

Universidad Mesoamericana, Oaxaca, México

\section{1.- INTRODUCCIÓN.}

En contextos educacionales el principal reto con el que se encuentran las innovaciones se refiere a los procesos de adopción por parte de las personas, los grupos y las instituciones. Para lograr experiencias exitosas se requiere tanto de participación activa y motivación de los agentes de cambio. A la hora de identificar los actores y determinar el protagonismo que tienen en el desarrollo de las innovaciones, los académicos resultan al final los actores centrales. En este contexto resulta de interés poder disponer de un diagnóstico de los factores que afectan la implementación de las tecnologías informacionales en los docentes, actividad que ayudaría a distinguir las necesidades formativas de los profesores en su etapa inicial y a nivel continuo.

El estudio de las etapas de adopción de la tecnología nos podría dar luz respecto de las características personales de los docentes además de la visión que tienen ellos de los medios tecnológicos en contextos educativos, fase que se considera previa a cualquier intento institucional de integrar los medios tecnológicos al currículum (Sánchez, 2003).

A continuación, en base al análisis de los modelos de adopción tecnológica que se encuentran en la literatura, se propone un modelo que intenta por un lado dinamizar los enfoques que ven la adopción tecnológica como niveles "graduados” y por otro lado proporcionar una conceptualización más nítida de las acciones o conductas hacia la adopción de los medios tecnológicos. Esto último tiene fuertes implicaciones metodológicas tanto en la definición de las variables como en la operacionalización de las mismas.

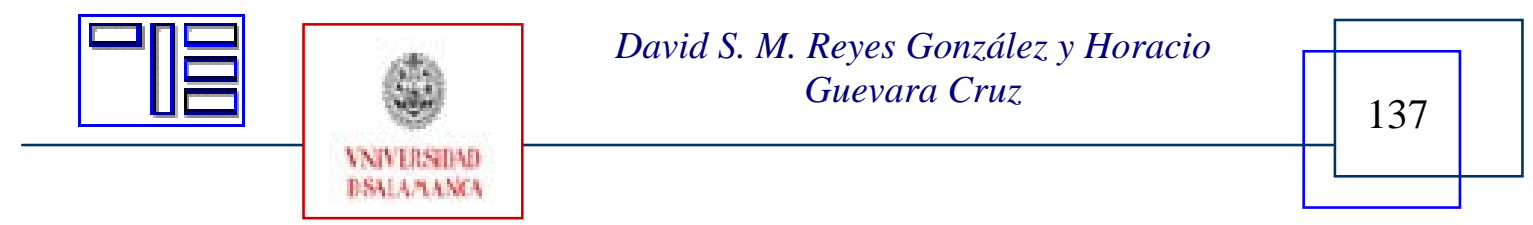


Revista Electrónica Teoría de la Educación.

Educación y Cultura en la Sociedad de la Información.

http://www.usal.es/teoriaeducacion

Vol. 10. No 1. Marzo 2009

\section{2.- ADOPCIÓN DE LOS MEDIOS TECNOLÓGICOS.}

En la literatura se han propuesto distintos esquemas o modelos que abordan el modo en que un profesor adopta una innovación tecnológica. Existe acuerdo en que ésta se desarrolla en niveles o etapas crecientes de adopción desde un punto inicial hasta un estadio final donde los medios tecnológicos se articulan en la práctica educativa haciéndola parte del currículum (J Sánchez, 2003). El desarrollo estaría marcado por niveles de creciente autonomía de búsqueda, uso, experimentación y acercamiento siempre regulado por las concepciones y creencias pedagógicas y epistemológicas y en base al análisis de la información que dispone.

Si el objetivo de una Institución Educativa es lograr una efectiva integración de la tecnología en el currículum, se hace necesario determinar los factores que afectan la implementación tecnológica de manera que se puedan desarrollar políticas conducentes a favorecer e intentar comprender el comportamiento de los docentes frente a los medios tecnológicos.

\subsection{Se comienza intruseando.}

Las propuestas de adopción tecnológica (tabla 1) en su mayoría comienzan desde un nivel fundamental caracterizado por una total carencia de conocimientos tecnológicos hasta que, luego de una alfabetización digital, logran adquirir las habilidades y técnicas que les permiten realizar prácticas educativas mediadas por la tecnología.

\begin{tabular}{|c|c|c|c|c|c|}
\hline $\begin{array}{l}\text { (Dwyer, Rings- } \\
\text { taff, \& Sand- } \\
\text { holtz, 1991) }\end{array}$ & $\begin{array}{l}\text { (Mandinach } \\
\text { \& Cline, } \\
\text { 1994) }\end{array}$ & (Rogers, 1995) & $\begin{array}{l}\text { (Romagno- } \\
\text { li, Femen- } \\
\text { ías, \& Con- } \\
\text { te, 1999) }\end{array}$ & $\begin{array}{c}\text { Sánchez } \\
\text { (2003) }\end{array}$ & Noon $(2005)^{[1]}$ \\
\hline Entrada & Supervivencia & Conocimiento & Inicio & Apresto & Prealfabetización \\
\hline Adopción & Maestría & Persuasión & Adaptación & Uso & Tecnocrático \\
\hline Adaptación & Impacto & Decisión & $\begin{array}{l}\text { Apropia- } \\
\text { ción }\end{array}$ & Integración & Tecnotradicionalista \\
\hline Apropiación & Innovación & Implementación & Innovación & & $\begin{array}{c}\text { Tecnoconstructivis- } \\
\text { ta }\end{array}$ \\
\hline Invención & & Confirmación & & & \\
\hline
\end{tabular}

Tabla 1. Modelos de Adopción Tecnológica presentes en la literatura

Como una extensión del modelo propuesto por los investigadores del proyecto Apple Classrooms of Tomorrow (ACOT) (Dwyer, Ringstaff, \& Sandholtz, 1990; Dwyer et al., 1991), Mandinach y Cline (1994) denominan supervivencia a la etapa inicial de difusión de la tecnología en la escuela. En ella los docentes "lucharían” por aprender el funcio-

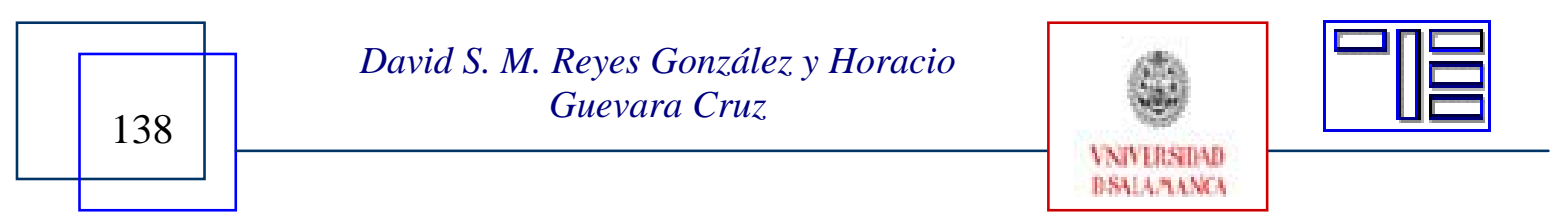


namiento de los medios tecnológicos sobre todo mediante pruebas de ensayo-error. Del mismo modo, Russell (1990), tomando al correo electrónico como base de análisis, propone que el aprendizaje adulto atraviesa 6 fases de integración tecnológica. Comenzando con sentimientos de temor y desconcierto, Russell plantea que el nivel inicial conocimiento se caracteriza por la presentación de la información y el comienzo de los posibles usos de la herramienta. Estos enfoques asumen que las TI no son más que una destreza operativa que se enseña, y así se establece el marco propicio para plantear la alfabetización digital desde cero sin considerar algún tipo de conocimientos o experiencias previas.

En la actualidad las TI son un medio que propicia la autoformación, de ahí que en cierto grado, determina su utilización (Amar, 2006) . En efecto, los profesores poseen cierto nivel de habilidades en TI y traen consigo grados de dominio técnico cada vez mayores, ya sea adquirido como parte de su formación inicial o a través de experiencias personales (OCDE, 2003). En éste último caso, en un número considerable de docentes, la motivación de implementar las TI en el aula surge a partir de si propia profesionalidad (J. Sánchez \& Salinas, 2008). En su gran mayoría los docentes universitarios manejan tarjetas de transacciones bancarias, un teléfono móvil, una cuenta de correo electrónico, espacios de acción que necesariamente los incluye en una dinámica relacional con la tecnología que no corresponde a la ausencia de conocimientos tecnológicos y los sitúan dentro de la cotidianidad tecnológica que contempla que el uso de las TIC es un requisito para la participación en la sociedad de la información y el conocimiento (Guevara Cruz, 2006).

Sin que el sistema de formación tradicional lo haya advertido, fuera del aula, algunos profesores hemos intruseado con la tecnología. Por ello, y coincidiendo con la propuesta de Noon para un primer nivel de conocimientos mínimos (McKenzie, 2005b), se estima conveniente considerar un primer nivel de adopción tecnológica de intrusión en el que tod@s los profesores poseen -aunque sea en un grado mínimo- conocimiento de las TI. No se puede continuar considerando que los docentes sean una "página en blanco" sobre la cual hay que trazar competencias o habilidades relacionadas con el uso de tecnologías infocomunicacionales. Se debe pasar de una alfabetización digital a una implementación efectiva de la TIC y dejar de proporcionar instrucciones operativas. En este sentido, Robalino y Corner (2005) consideran que la alfabetización digital es una primera etapa que retrasa el "uso pertinente de las TIC y de su incorporación a la didáctica propiamente tal” (Robalino \& Körner, 2005, 27).

Con excepción del enfoque tecnoconstructuvista (McKenzie, 2005a), el resto de los modelos orientan la adopción de la tecnología hacia la actualización de contenidos, métodos y materiales. Esta forma de abordar la educación en tecnologías informacionales comete el error de interpretar los espacios virtuales de formación como escenarios tradicionales fuertemente escolarizados.

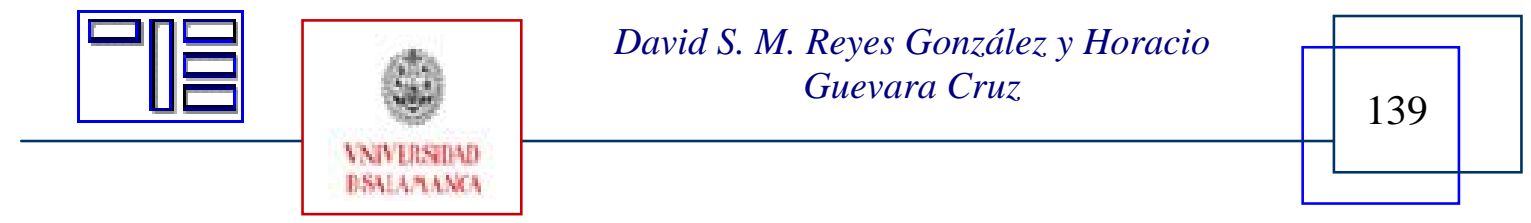


La condición necesaria para que los modelos basados en la insuficiencia y obsolescencia de conocimientos por parte de los profesores reorienten su posición, se halla en la concepción de la consecuencia de la dinámica relacional que se establece entre el docente y la tecnología informacional. La emergencia de modelos pedagógicos basados en la conformación de comunidades de práctica (Mayes \& de Freitas, 2004) indica que la forma de aprender de los docentes está cambiando en lo referente al uso de las TI y en los procesos sociales interactivos (De Freitas, 2006). Estos cambios implican no solamente un incremento en la flexibilidad del aprendizaje, sino que también en las infraestructuras y los sistemas sociales que soportan estos procesos(Guevara Cruz \& Reyes González, 2007).

\section{3.- AMBIGÜEDAD DE TÉRMINOS.}

Es común hablar de adopción, apropiación, adaptación o de implementación para conceptualizar el "uso" que hacen los docentes de los medios tecnológicos. Muchas veces en las investigaciones se opta por uno de los conceptos asociados a la integración de las TI en el currículum sin reconocer la complejidad que yace bajo un término tan genérico como puede ser "el uso" de las TIC (Scrimshaw, 2004). Con base en el argumento de adopción propuesto por Rogers (1995) en esta sección se pretende estructurar un marco de referencia para poder diferenciar claramente lo que es uso, adopción, utilización, apropiación.

\subsection{Implementación.}

Resulta interesante considerar el concepto de implementación de Rogers por dos razones fundamentales. La primera corresponde a la clara delimitación de conductas que Rogers propone; la fase de implementación comienza sencillamente cuando "se pone en uso" la innovación y culmina cuando las operaciones con la innovación comienzan a institucionalizarse o se regularizan. Un segundo aspecto que resulta atrayente es que en la implementación es la primera vez que el individuo pone en práctica la innovación. De acuerdo con el autor, en las fases iniciales del proceso de adopción, los individuos sólo se relacionan con la innovación mediante ejercicios mentales cognitivos. Al conocer una determinada innovación el docente se cuestiona: “qué es esa innovación y cómo funciona”, e inmediatamente surgen pensamientos de tipo afectivo/evaluativo con el objeto novedoso. En base a nuestros sistemas de creencia comenzamos a formar una actitud hacia nuestra práctica con el objeto en diversos contextos, ¿qué consecuencias (positivas o negativas) tendría incorporar un medio tecnológico en mis prácticas educativas? o ¿qué piensan mis pares o mi circulo social próximo sobre que yo incorpore un medio tecnológico novedoso a mis clases?. Pero no es sino en la implementación cuando ocurre la implicación práctica del profesor. Los procesos mentales no ocurren con el individuo aislado de su contexto sino que emergen ahora con el individuo actuando con el instrumento, poniendo en marcha o implementando las TIC en contextos educativos específicos.

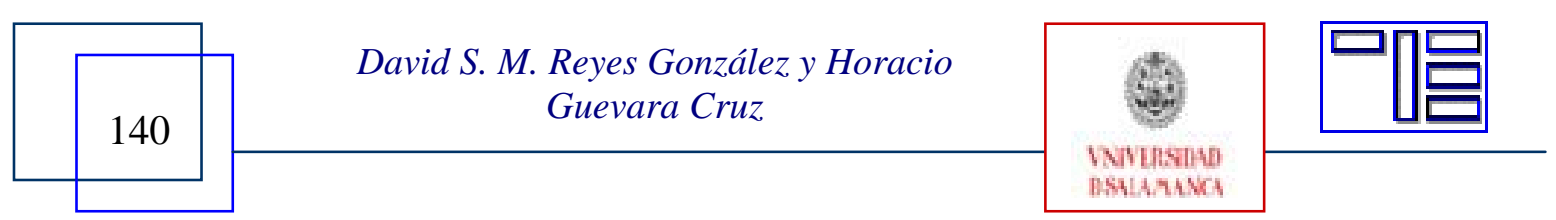


Entendemos por implementación de las TI en contextos educativos la puesta en marcha de la tecnología en su sistema de enseñanza habitual. En esta fase el docente ejecuta acciones relacionadas con la tecnología; cambia de la pizarra a la presentación digital, de la fotocopia a las referencias de Internet, incluso puede ser que ocupe con sus estudiantes recursos Web para apoyar sus clases, condicionado por la disponibilidad de recursos tanto subjetivos (sistemas afectivos, cognitivos, de personalidad, etc.) como objetivos (infraestructura, comercialización, económicos, etc.). El profesor comienza a relacionarse con las TI en sus prácticas y como resultado surgen los primeros imprevistos con la tecnología, ya que aún existe cierto grado de incertidumbre sobre las consecuencias de su implementación. Estas dudas tienen una implicación marcada en la forma en que implementan la innovación; el profesor pasa de un adoptante pasivo a uno activo y se implica con los medios tecnológicos hasta el punto en que puede modificarlos y dotarlos de un matiz personal en la forma en que se implementan. Esto posiblemente se debe a que el resultado de la interacción docente/TIC no está determinado por las propiedades de los medios tecnológicos sino de cómo ellos son vistos o tomados por los profesores al incorporarlos a su dinámica.

Esta fase de experimentación con la tecnología resulta crucial en el proceso, ya que esto puede llevar al docente o bien a involucrarse cada vez más en las herramientas tecnológicas o bien retroceder a espacios más seguros donde se sienta más hábil en su manejo. En caso de abandonar la práctica su regreso no será a cero, pues existe la experiencia de lo ya vivido. Más aun, la fase de implementación favorece la construcción de nuevas prácticas a través de la experimentación y la reflexión (Zhao, Frank, \& Ellefson, 2006).

\subsection{Integración Curricular.}

La simple comprensión y empleo de las TIC no garantiza un uso efectivo en los procesos formativos, ya que se deben respaldar sobre unos principios metodológicos y didácticos que las justifiquen y delimite. Tal como afirma Lacruz "un cambio en la educación no es posible sin un cambio en los currículum y en las actitudes y aptitudes del profesorado frente a las exigencias que exigen los procesos de reforma" (Lacruz Alcocer, 2002). Por ello, se cree necesario, dentro del plan de formalizar un proceso de incorporación de los medios por los sistemas de formación, que las TIC se integren en las estrategias educativas evitando que se conviertan en prácticas insustanciales o que ocurran por accidente. Se puede considerar entonces que la integración de las TIC al currículum corresponde al nivel de logro último que debemos conseguir, el estado de acción donde el docente construye, utiliza y evalúa las tecnologías.

Tal como hemos afirmado anteriormente, las acciones educativas mediadas por las nuevas tecnologías posibilitan la emergencia de nuevos paradigmas educativos, de procesos de aprendizaje más personalizados y flexibles. Esto tiene efectos directos tanto en la selección de los contenidos curriculares como en la metodología, "algo que condicionará los diferentes tipos de integración curricular” (Amar Rodríguez, 2006). Al respecto Noon plantea que el nivel más alto de integración de la tecnología se alcanza en lo que

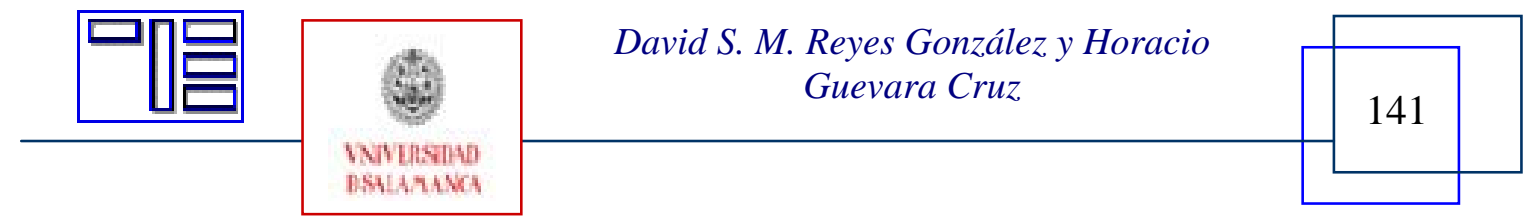


él denomina tecnoconstructivismo. En este estado los profesores utilizan la tecnología como medio para modificar y transformar los espacios educativos tradicionales en nuevos y diferentes ambientes de aprendizaje para los estudiantes. Una vez que permitimos que se aclare el conflicto entre nuestras creencias epistemológicas, nuestras creencias pedagógicas y el uso que le damos a la tecnología, es posible que se aprecien las posibilidades que ellos permiten para nuestros estudiantes. Una integración significativa de las TIC que supere este nivel umbral supone el cambio de las convicciones del profesorado y es, inevitablemente un proceso complejo que exige mucho tiempo (Niederhauser \& Stoddard, 2001).

En definitiva se plantea la integración curricular tecnoconstructivista como el nivel al que debemos llegar cuando adoptamos las TI con fines educativos. Lo interesante de esta propuesta se encuentra en que saber que hemos logrado ser un tecnoconstructivista es un juicio subjetivo, pues la forma en que podemos utilizar y dar sentido a la tecnología evolucionan constantemente hacia nuevos campos; recursos web 2.0, correo electrónico, trabajo colaborativo, viajes virtuales, blogs, flogs, presentaciones multimedia, RSS, etc., nos recuerdan que disponemos de herramientas que aún no acaban de mostrarnos el verdadero potencial que podemos alcanzar en las mediaciones comunicacionales entre individuos.

\section{4.- TRANSICIÓN ENTRE NIVELES.}

El enfoque de etapas o escalafones que gobierna los modelos de adopción tecnológica, nos sugiere que existen momentos de tránsito entre los niveles; momentos en que un docente manifiesta evolución tanto en sus acciones como en sus procesos mentales consiguiendo una transición. ¿Qué factores resultan críticos en la transición entre un nivel y otro? ¿Cómo ocurre el recorrido desde la intrusión hacia la integración curricular?

\subsection{Actitud.}

En un estudio internacional sobre las TIC y el cambio organizativo en las escuelas realizado por la OECD (Pont, 2000) se seleccionaron 94 buenas prácticas en 23 países del mundo elegidos por sus innovaciones y si inversión en TIC. El estudio sostiene que los ritmos de adopción de las TIC en las escuelas siguen los ritmos tradicionales de difusión de innovaciones (innovation-decision process), afectados principalmente por las características personales de los innovadores (Venezky, Davis, \& OECD/CERI, 2002). El modelo de Rogers ha sido experimentado en muchas ocasiones, y en la mayoría de ellas los sujetos reconocen haber pasado por las etapas del proceso de decisión-innovación. Aunque determinar los procesos mentales intrapersonales no sea un asunto trivial, "the evidence is most clear-cut for the knowledge and decision stages and somewhat less so for the persuasion stage. Only rather poor data are available on the distinctiveness of the implementation and confirmation stages" (Rogers, 1995).

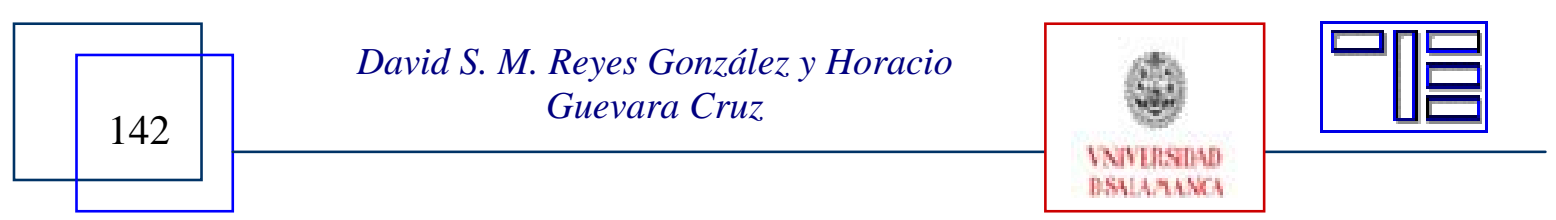


De acuerdo con Rogers luego de enterarse que existe una innovación (conocimiento), el docente pasa por una fase en que reúne información suficiente para formarse una actitud hacia el objeto de innovación (persuasión). Luego en base a sus creencias, evalúa las características de la innovación y su posible afiliación en ella (decisión). Posterior a estas actividades mentales el docente pasa a una fase de acción directa con la innovación (implementación); el profesor pasa de un adoptante pasivo a uno activo y se implica con la innovación hasta el punto en que puede reinventar la innovación datándola de un matiz personal en la forma en que se implementa.

Otros diversos estudios confirma la tesis de Roger de que las actitudes de los docentes juegan un papel decisivo en la integración de las TI (Albirini, 2006; Kitchen, Butt, Mackenzie, \& Finch, 2006; C. P Lim, 2007; Sugar, Crawley, \& Fine, 2004). Sin embargo, estos planteamientos resultan insuficientes, pues, como señalan algunos autores (Hennessy, Ruthven, \& Brindley, 2005; Scrimshaw, 2004) puede ocurrir que la introducción de las nuevas tecnologías en la enseñaza dependa, sobre todo, de determinadas percepciones y creencias que tienen los profesionales, ya sea de tipo epistemológico o metodológico, sobre las nuevas tecnologías.

La visión de que las creencias pedagógicas provocan resistencia al cambio provocando que persistan las prácticas instruccionales didácticas (C. P. Lim \& Chan, 2007; Niederhauser \& Stoddard, 2001) nos remite a considerar tres aspectos en concreto para el análisis de los factores que afectan la efectiva integración curricular de las TIC. Por un lado el factor personal que avalúa la afiliación en prácticas mediadas por la tecnología, un factor contextual o social que ejerce presión sobre las decisiones que tomamos y un factor de control sobre lo que creemos ser capaces hacer o de saber utilizar y nos provoca una percepción de que llevar a cabo una conducta sea fácil o dificultoso.

En este sentido, la Teoría del Comportamiento Planificado (Ajzen, 1985, 1991; Ajzen \& Madden, 1986) (TPB de la voz inglesa Theory of Planned Behavior) emerge como una formulación teórica atractiva, ya que por un lado aporta resultados favorables respecto de otros modelos teóricos en el ámbito de la comprensión de la conducta de adopción de las TIC por parte de los profesores (Lim, Gan, \& Chan, 2002; Reyes González, 2007) y, por otra parte -como se explica más adelante-, cada uno de los constructos integra elementos fundamentales para la comprensión de los determinantes de las conductas asociadas con el uso de la tecnología por las personas.

En términos generales TPB (ver figura 1) considera que la acción humana está dirigida por tres tipos de consideraciones: creencias sobre las probables consecuencias o sobre otras características de la conducta (creencias conductuales); creencias respecto de las expectativas normativas de otras personas (creencias normativas); y creencias sobre la presencia de factores que pueden fomentar u obstaculizar el desarrollo de una conducta (creencias de control). En sus respectivos agregados, las creencias conductuales producen una actitud hacia la conducta favorable o desfavorable; las creencias normativas, por su parte, forma una visión subjetiva de las presiones sociales o normas subjetivas; y

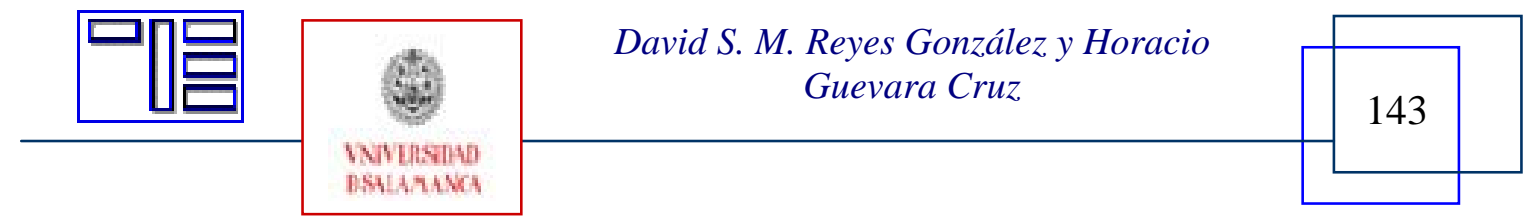


Revista Electrónica Teoría de la Educación.

Educación y Cultura en la Sociedad de la Información.

http://www.usal.es/teoriaeducacion

Vol. 10. No 1. Marzo 2009

las creencias de control generan en el individuo una percepción de que llevar a cabo una conducta sea fácil o dificultoso (control conductual percibido). En combinación, la actitud hacia la conducta, la norma subjetiva y el control conductual percibido permiten la formación de una intención conductual (o simplemente intención).

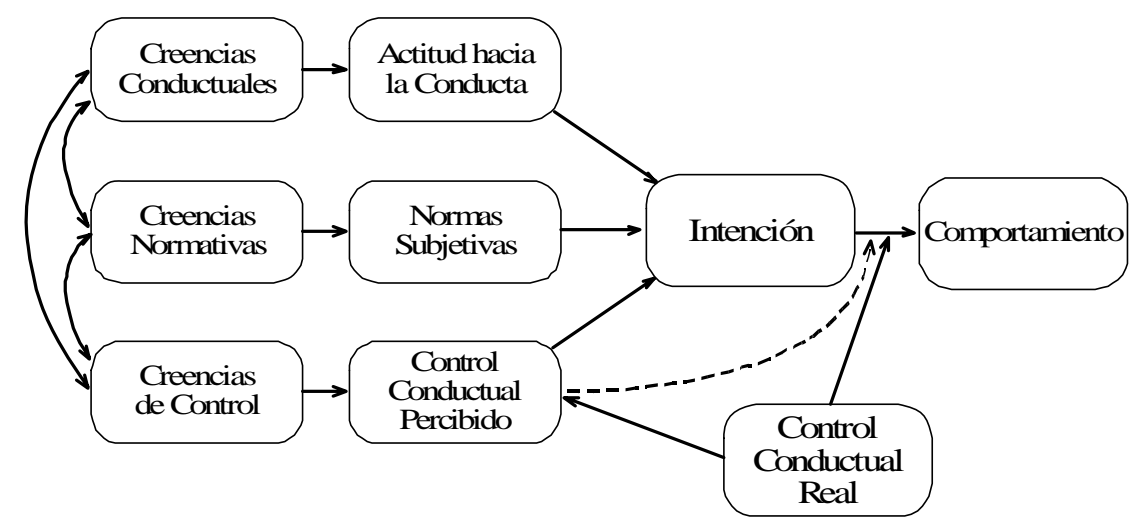

Figura 1. Teoría del Comportamiento Planificado ${ }^{[2]}$

Es nuestro interés emplear el modelo de la Teoría del Comportamiento Planificado como un marco conceptual para desarrollar un estudio que examine las variadas influencias que permiten a un docente pasar de la fase de intrusión hacia la implementación. Por otra parte, el modelo de Fishbein y Ajzen ha sido utilizado para estudiar los factores que permiten a los docentes adoptar las tecnologías en los procesos educativos (Cox, Preston, \& Cox, 1999a, 1999b; Chen \& Chen, 2006; Sugar et al., 2004), y esta variedad de estudios que lo emplean nos permite realizar comparaciones, contrastar y/o corroborar resultados.

De entre todos los constructos propuestos por TPB, los resultados nos indican que tanto la el componente normativo como el de control muestran un efecto menor en la motivación de los docentes a implementar las nuevas tecnologías. Sólo la actitud hacia la conducta predijo de mejor manera la intención de los docentes a implementar sistemas informacionales en sus prácticas académicas (Sugar et al., 2004). Por lo que podemos plantear la siguiente hipótesis: un docente debe formarse una actitud positiva hacia las nuevas tecnologías para lograr vencer la barrera que le impide adoptar estrategias educativas mediadas por las tecnologías. Así, la actitud se convierte en un factor psicológico transitivo entre la intrusión y la implementación de las TIC en el aula.

\subsection{Apropiación.}

La fase que hemos denominado como implementación corresponde a una fase de experimentación, las representaciones mentales no se dan con el individuo aislado sino que emanan con el individuo actuando con el instrumento tecnológico, poniendo en marcha o implementando las TIC en el aula. Esto implica que su sistema de pensamiento comienza a percibir que la tecnología le permite nuevos patrones de acción educativa, de

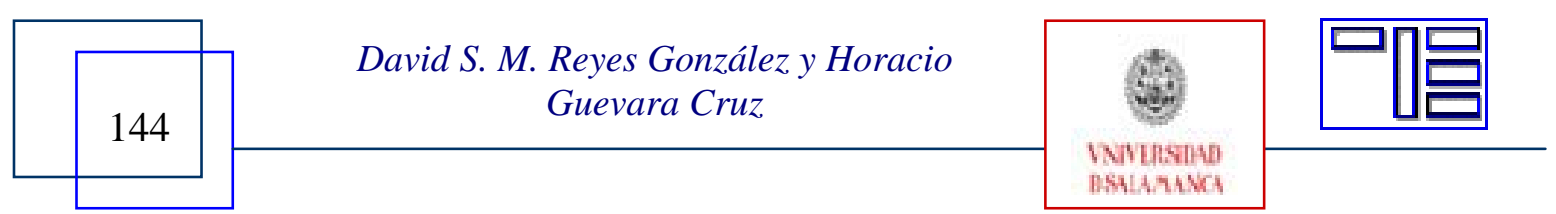


nuevos espacios, con sus consecuencias en la productividad de la clase, al modificar su rol de poseedor del contenido de la asignatura a un facilitador de la construcción del conocimiento con el estudiante. Esta conciencia de las necesidades formativas de la sociedad se genera únicamente cuando el docente permite que las tecnologías operen en contextos formativos. Cuando el ordenador se introduce en el aula es que somos capaces de percibir que las tecnologías no son neutras, tienen falencias y virtudes, por ello vamos probando nuevas estrategias y métodos que nos conducen hacia la invisibilidad del ordenador (Gros Salvat, 2000), los sistemas tecnológicos que utilizamos ya no son el fin sino que nos esforzamos en lograr el acoplamiento de estos productos con los objetivos educativos. De acuerdo con Begoña Gros "lo que se trata es de apropiarse de la herramienta, de utilizarla de manera más conveniente desde el punto de vista educativo." (Gros Salvat, 2000). En este sentido se considera que la apropiación constituye un segundo factor de transito entre la fase de implementación y la integración curricular.

El concepto de apropiación que manejamos es muy similar al propuesto por los investigadores del proyecto ACOT (Dwyer et al., 1990), sin embargo nuestro enfoque aborda el proceso de adopción tecnológica por parte de los docentes desde una perspectiva dinámica, ya que junto con la actitud hacia la implementación de las TIC en el aula (o simplemente actitud) la apropiación es un factor de tránsito para dos etapas de acción como son la implementación de las TIC y la integración curricular tecnoconstructivista (figura 2).

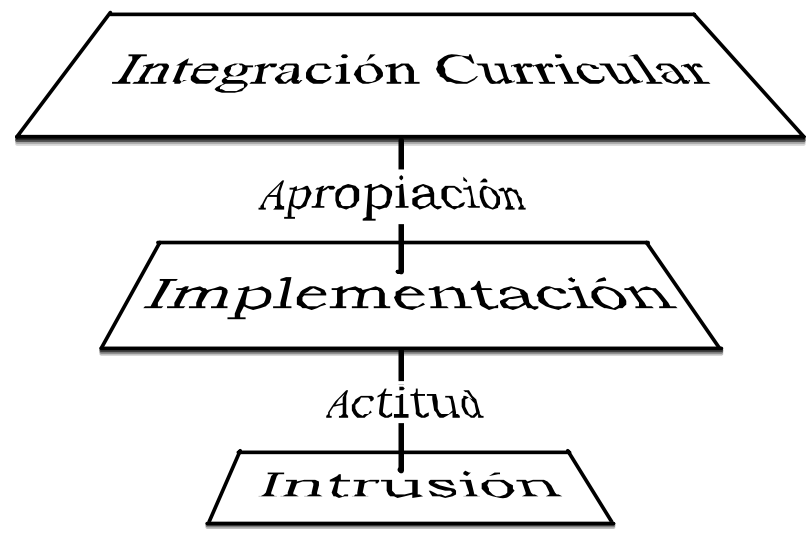

Figura 2. Modelo de Adopción Dinámico de las TIC por los docentes

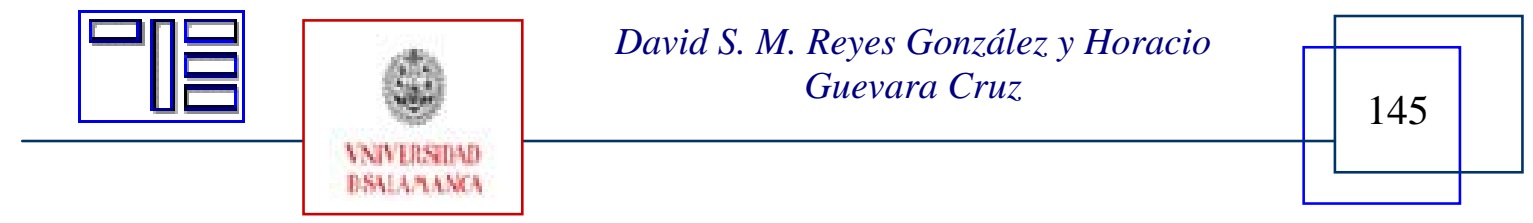




\section{5.- CONCLUSIÓNES.}

A lo largo del texto se ha tratado de dar una estructura teórica actual a los enfoques tradicionales de adopción tecnológica. Se pudo reconocer la existencia de factores internos transitivos que nos permitiría dar cuenta de cómo sería el paso de un nivel de adopción a otro, dinamizando el punto de vista tradicional de adopción tecnológica por niveles. La diferencia entre comportamientos que denominamos acciones y los factores de tránsito de carácter psicológico entre estos ha sido reconocida anteriormente por los investigadores del proyecto ACOT (Dwyer et al., 1990 y 1991). Ellos asumían que la evolución de las prácticas docentes con sistemas informáticos ocurría gradualmente a medida que sus creencias como sus prácticas eran reemplazadas por otras nuevas. Es por ello que Niederhauser no duda en reconocer el trabajo de ACOT como "the only major study to examine the relationships among teachers' epistemological beliefs, pedagogical beliefs, and their instructional uses of technology” (Niederhauser \& Stoddard, 2001).

Lo siguiente es probar este modelo, estudiar si efectivamente existen estos factores internos que lograrían la promoción de un nivel de adopción a otro. Se cree que la Teoría del Comportamiento Planificado es una estructura teórica que permitiría indagar en estas fases transitivas pues contempla factores actitudinales, normativos y de control que han sido probados en estudios similares.

\section{6.- REFERENCIAS.}

AJZEN, I. (1985): From intentions to actions: A theory of planned behavior. En J. Kuhl \& J. Beckman (Eds.), Action-control: From cognition of behavior (pp. 11-39). Heidelberg: Springer.

AJZEN, I. (1991): The theory of planned behavior. Organizational Behavior and Human Decision Processes, 50, 179-211.

AJZEN, I., \& MADDEN, T. J. (1986): Prediction of goal-directed behavior: attitudes, intentions, and perceived behavioral control. Journal of Experimental Social Psychology, 22, 453-474.

ALBIRINI, A. (2006): Teachers' attitudes toward information and communication technologies: the case of Syrian EFL teachers. Computers \& Education, 47(4), 373-398.

AMAR RODRÍGUEZ, V. (2006): Nuevas tecnologías y medios de comunicación en la educación. Cádiz, Universidad de Cádiz.

COX, M., PRESTON, C., \& COX, K. (1999a): What factors Support or Prevent Teachers from Using ICT in their Clasrrom. Paper presentado en British Educational

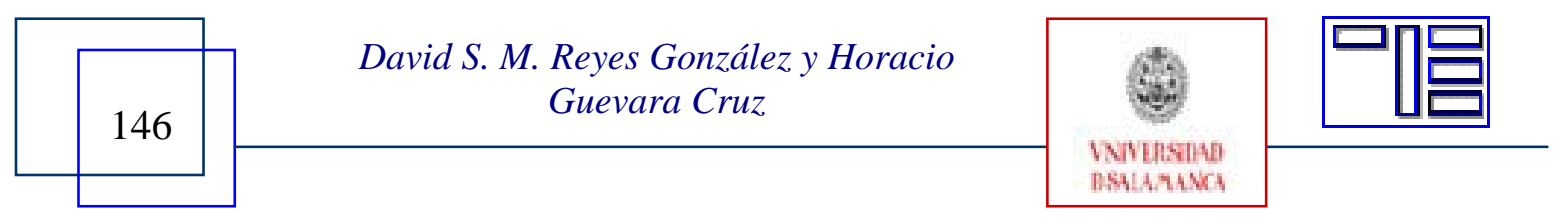


Research Association Annual Conference. University of Sussex at Brighton. Extraído en Agosto del 2007.

http://www.leeds.ac.uk/educol/documents/00001304.htm.

COX, M., PRESTON, C., \& COX, K. (1999b): What Motivates Teachers to use ICT? Paper presentado en British Educational Research Association Annual Conference. University of Sussex at Brighton. Extraído en Agosto del 2007, desde www.leeds.ac.uk/educol/documents/00001329.htm.

CHEN, T., \& CHEN, T. (2006): Examination of attitudes towards teaching online courses based on theory of reasoned action of university faculty of Taiwan. British Journal of Educational Technology, 37(5), 683-693.

DE FREITAS, S. (2006): Collaborative e-support for life long learning. British Journal of Educational Technology, 37(6), 683-693.

DWYER, D. C., RINGSTAFF, C., \& SANDHOLTZ, J. H. (1990): The Evolution of Teachers' Instructional Beliefs and Practices in High-Access-to-Technology Classrooms: First-Fourth Year Findings (No. 8). California.

DWYER, D. C., RINGSTAFF, C., \& SANDHOLTZ, J. H. (1991): Changes in teachers' beliefs and practices in technology-rich classrooms. Educational Leadership, 48(8), 45-52.

GROS SALVAT B. (2000): El ordenador invisible. Hacia la apropiación del ordenador en la enseñanza. Barcelona, Gedisa.

GUEVARA CRUZ, H. (2006): Cuando hablamos de cotidianidad tecnológica. Paper presentado en III Congreso Online-Observatorio para la Cibersociedad. Conocimiento Abierto. Sociedad Libre. Extraído en Agosto del 2007, desde http://www.cibersociedad.com/congres2006/gts/comunicacio.php?id=731\&lleng ua $=$ es.

GUEVARA CRUZ, H., \& Reyes González, D. S. M. (2007): La cultura de la escuela ante la contingencia de las nuevas tecnologías. La reconfiguración del docente ante el sentido de educar actual. Paper presentado en Seminario en modalidad presencial y Online. Salto, Uruguay. Extraído en Agosto del 2007, desde http://grupointernacionaltic.org/.

HENNESSY, S., RUTHVEN, K., \& BRINDLEY, S. (2005): Teachers perspectives on integrating ICT into subject teaching: Commitment, constrains, caution and change. Journal of Curriculum Studies, 37(2), 155-192.

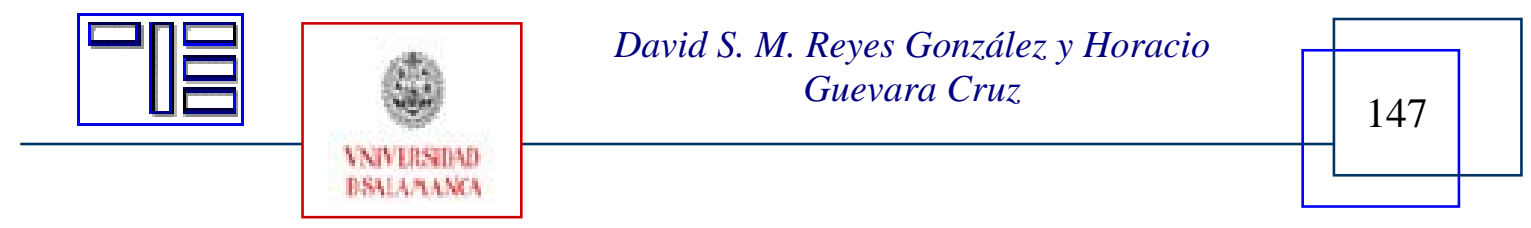


KITCHEN, S., BUTT, S., MACKENZIE, H., \& FINCH, S. (2006): Curriculum Online Evaluation: Report of the third survey of schools: Becta ICT Research.

LACRUZ ALCOCER, M. (2002): Nuevas tecnologías para futuros docentes. Toledo, Ediciones Universidad de Castilla-La Mancha.

LIM, C. P. (2007): Effective integration of ICT in Singapore schools: pedagogical and policy implications. Educational Technology Research and Development, 55(1), 83-116.

LIM, C. P., \& CHAN, B. C. (2007): microLESSONS in teacher education: examining pre-service teachers’ pedagógical beliefs. Computers \& Education, 48(3), 474494.

LIM, J., GAN, B., \& CHAN, T.-T. (2002): A survey on NSS adoption intention. Paper presentado en 35th Annual Hawaii International Conference on System Sciences, Hawaii.

MANDINACH, B., \& CLINE, F. (1994): Classroom Dynamics: Implementing a Technology-based Learning Enviroment. Nueva York, Lawrence Erlbaum Associates.

MAYES, T., \& de FREITAS, S. (2004): Review of e-learning theories, frameworks and models: Joint Information Systems Committee (JISC).

MCKENZIE, W. (2005a, Agosto 2007): Becoming a Technoconstructivist. On Cue, Spring 2005, 21-23.

McKENZIE, W. (2005b): Multiple Intelligences and Instructional Technology. Extraído en Agosto del 2007, desde www.iste.org.

NIEDERHAUSER, D. S., \& STODDARD, T. (2001): Teachers’ instructional perspectives and use of educational software. Teaching and Teacher Education, 17(1), 15-31.

OCDE. (2003): Los Desafíos de las Tecnologías de la Información y las Comunicaciones en la Educación. Madrid, OCDE/CERI.

PONT, B. (2000): La integración de las TICs en el proceso educativo: Una visión internacional. Paper presentado en Seminario Internacional de la Red Enlaces, Santiago de Chile.

REYES GONZÁLEZ, D. S. M. (2007): LaTeoría del Comportamiento Planificado. Trabajo de Investigación no publicado. Universidad de Salamanca.

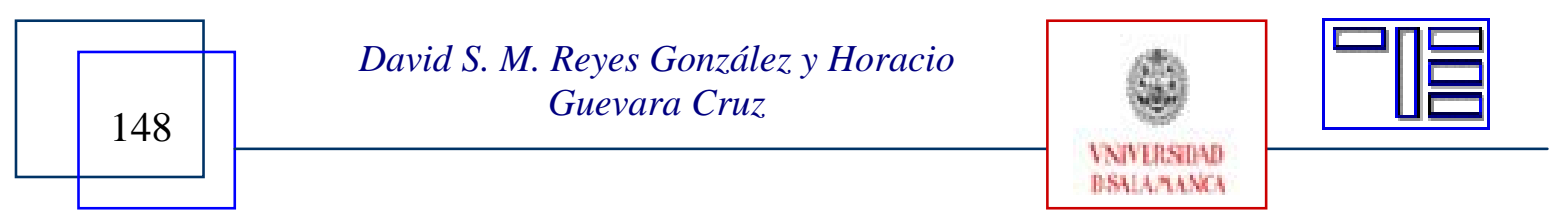


ROBALINO, M., \& KöRNER, A. (2005): Formación Docente y las Tecnologías de Información y Comunicación. Santiago de Chile: OREALC/UNESCO.

ROGERS, E. (1995): Diffusion of Innovations. Nueva York, The Free Press.

ROMAGNOLI, C., FEMENÍAS, G., \& CONTE, P. (1999): Internet, un nuevo recurso para la educación. Chile, MINEDUC.

RUSSELL, A. (1990): Electronic mail: Is it intrusive or invisible? En J. G. Hedberg, J. Steele \& M. Mooney (Eds.), Converging Technologies: Selected papers from EdTech'90 (pp. 108-115). Canberra: AJET Publications.

SÁNCHEZ, J. (2003): Integración curricular de las TICs. Conceptos y modelos [Versión Electrónica]. Revista Enfoques Educacionales, 5, 51-65. Extraido en Agosto del $2007 \quad$ desde http://csociales.uchile.cl/publicaciones/enfoques/07/Sanchez_IntegracionCurricu larTICs.pdf.

SÁNCHEZ, J., \& SALINAS, A. (2008): ICT \& learning in Chilean schools: Lessons learned. Computers \& Education, 51, 1621-1633.

SCRIMSHAW, P. (2004): Enabling teachers to make successful use of ICT. Extraído en Agosto del 2007, desde http://partners.becta.org.uk/uploaddir/downloads/page_documents/research/enablers.pdf.

SUGAR, W., CRAWLEY, F., \& FINE, B. (2004): Examining teachers' decisions to adopt new technology. Educational Technology and Society, 7(4), 201-213.

VENEZKY, R. L., DAVIS, C., \& OECD/CERI. (2002): Quo Vademus?The transformation of schooling in a networked world [Versión Electrónica]. Extraido en Agosto del 2007 desde http://www.oecd.org/dataoecd/48/20/2073054.pdf.

ZHAO, Y., FRANK, K. A., \& ELLEFSON, N. C. (2006): Fostering meaningful teaching and learning with technology: Characteristics of effective profesional development. En E. Ashburn \& R. Floden (Eds.): Meaningful Learning Using Technology. What Educators Need to Know and Do. Nueva York, Teachers College Press.

\section{Notas:}

${ }^{[1]}$ Citado en (McKenzie, 2005b).

${ }^{[2]}$ Adaptado de http://www.people.umass.edu/aizen/index.html. Consultado Abril 2007.

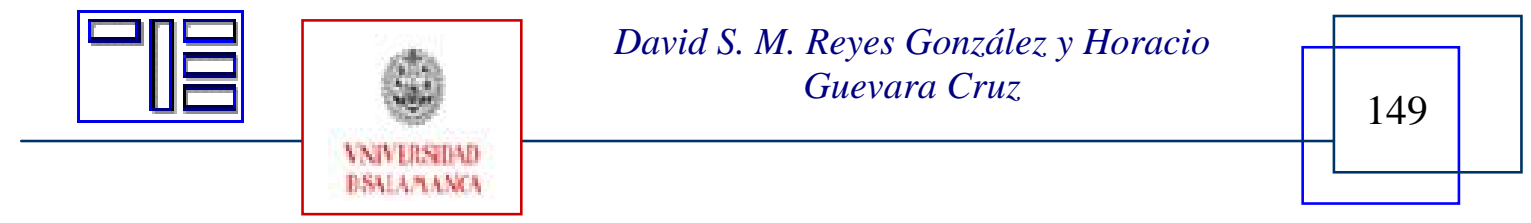


Revista Electrónica Teoría de la Educación.

Educación y Cultura en la Sociedad de la Información.

http://www.usal.es/teoriaeducacion

Vol. 10. No 1. Marzo 2009

Para citar el presente artículo puede utilizar la siguiente referencia:

REYES GONZÁLEZ, David S. M. y GUEVARA CRUZ, Horacio (2009). Adopción de las tecnologías infocomunicacionales (TI) en docentes: actualizando enfoques. En SAN MARTÍN ALONSO, A. (Coord.) Convergencia Tecnológica: la producción de pedagogía high tech [monográfico en línea]. Revista Electrónica Teoría de la Educación: Educación y Cultura en la Sociedad de la Información. Vol. 10, nº 1. Universidad de Salamanca [Fecha de consulta: $\mathrm{dd} / \mathrm{mm} / \mathrm{aaaa}$.

http://www.usal.es/ teoriaeducacion/rev_numero_10_01/n10_01_reyes_guevara.pdf ISSN: 1138-9737 